\title{
A Trigeminal Schwannoma Masked by Solely Vestibulocochlear Symptoms
}

\author{
Lauran K. Evans $^{1} \quad$ Lazaro Peraza $^{1}$ Anthony Zamboni ${ }^{1}$ \\ ${ }^{1}$ Division of Otolaryngology, Department of Surgery, University of \\ Nevada, Reno School of Medicine, Reno, NV \\ Address for correspondence Lauran K. Evans, Division of \\ Otolaryngology, Department of Surgery, University of Nevada, \\ Reno School of Medicine, Reno, NV 89557 \\ J Am Acad Audiol 2020;31:449-454. \\ (e-mail: Lauranevans@gmail.com).
}

\begin{abstract}
Keywords

- acoustic neuroma

- medical error

- neurilemmoma

- trigeminal

- vestibulocochlear
\end{abstract}

\section{Introduction}

Schwannomas are benign tumors of Schwann cells that often arise within cranial nerves. More than $80 \%$ of intracranial schwannomas originate within the vestibulocochlear nerve, often causing slowly progressive high-frequency hearing loss, tinnitus, and vestibular dysfunction. This vestibulocochlear schwannoma is commonly referred to as an acoustic neuroma, which is actually a misnomer-it fails to recognize vestibular and Schwann cell involvement. Trigeminal nerve schwannomas occur less frequently $(<5 \%$ of intracranial schwannomas and $<0.3 \%$ of all intracranial tumors), often causing facial pain and numbness, but may also be asymp- tomatic. Trigeminal schwannomas are more commonly seen in women, and typically occur in middle-aged patients with an average age ofdiagnosis being 39 years (Arseni et $\mathrm{al}^{2}$; Agarwal ${ }^{1}$ ). The rarity of trigeminal schwannomas makes research and data on the condition scarce, with only case reports and case series published (Javalkar et $\mathrm{al}^{7}$; Sharma et $\mathrm{al}^{14}$; Zhang et $\mathrm{a}^{17}$; Fukaya and Yoshida ${ }^{6}$; Chowdhury et $\mathrm{al}^{4}$; Agarwal ${ }^{1}$ ). In fact, before the regular usage of computerized tomography (CT) and magnetic resonance imaging (MRI) scans, it was extremely difficult to properly diagnose trigeminal schwannomas. Treatment for this tumor includes watchful waiting, stereotactic radiosurgery, or surgical resection. Prognosis for these patients is hopeful, as the
Copyright $\odot 2020$ by the American Academy of Audiology. All rights reserved. Thieme Medical Publishers, Inc., 333 Seventh Avenue, New York, NY 10001 , USA.

Tel: +1(212) 760-0888.
DOI https://doi.org/ 10.3766/jaaa.19033. ISSN 1050-0545. 
benign tumor rarely conveys malignancy. Mass effect due to local invasion may occur as the schwannoma grows, resulting in hydrocephalus and compression of cranial nerves and brainstem structures, hence the trigeminal nerve symptoms (Rigamonti et $\mathrm{al}^{12}$ ).

Similar symptoms to a vestibulocochlear schwannoma occur from internal ear pathology, such as Meniere's disease. Meniere's disease is a clinical syndrome classified by episodic, spontaneous vertigo that is often associated with unilateral fluctuating sensorineural hearing loss, tinnitus, and aural fullness (Lopez-Escamez et $\mathrm{al}^{9}$ ). Meniere's disease can also cause low-or combined low- and high-frequency hearing loss, caused by what is thought to be excess endolymph in the labyrinthine system of the inner ear (Pearson and Brackmann $\left.{ }^{11}\right)$. The disease is more commonly unilateral but occurs in both ears in about $10 \%$ of cases (Sajjadi and Paparella ${ }^{13}$ ). Currently, there is no cure for Meniere's disease, but $>85 \%$ of patients who suffer from it show improvement with lifestyle changes and minimally invasive surgical procedures (Coelho and Lalwani ${ }^{5}$ ). There remains an overlap in symptomatology between middle/internal ear disorders with abnormalities in the vestibulocochlear neural pathway.

Thus, the rarity of trigeminal schwannomas, and the overlapping symptoms and presentation with other more common diseases, presents a challenge for clinicians. In these scenarios, it is increasingly important for clinicians to be aware of prior physician documentation and be particularly mindful to details to avoid a false diagnosis.

\section{Case Presentation}

\section{Initial Presentation}

A 48-year-old woman presented to an otolaryngologist with episodic left aural pressure, left-sided hearing loss, dizziness, disequilibrium, and nausea for one year, with progression over the last month. One year before, the patient had been referred to an outside otolaryngologist by her primary care physician for acute onset of left-sided tinnitus, left-sided hearing loss, and dizziness. She denied facial numbness, pain, or weakness. The otolaryngologist then ordered a brain MRI, with and without contrast, to evaluate for a suspected etiology of these symptoms, a vestibulocochlear schwannoma. However, no abnormalities were noted in relation to the vestibulocochlear nerve or brainstem nucleus. Instead, imaging showed a 6- $\times 8$-mm mass along the root of the left trigeminal nerve, and the patient was referred to radiation oncology (see - Figures 1 and 2). Radiologists and radiation oncologists concluded that her symptoms were unrelated to the incidental mass seen on imaging and diagnosed her with a trigeminal schwannoma.

\section{Initial Treatment}

It was discussed by the radiation oncologists whether watchful waiting, surgical removal, or stereotactic radiosurgery would be most appropriate for her incidental, asymptomatic trigeminal schwannoma. The option of watchful waiting would entail follow-up MRI scans every six months to one year, with treatment initiation if the tumor were to increase in size.

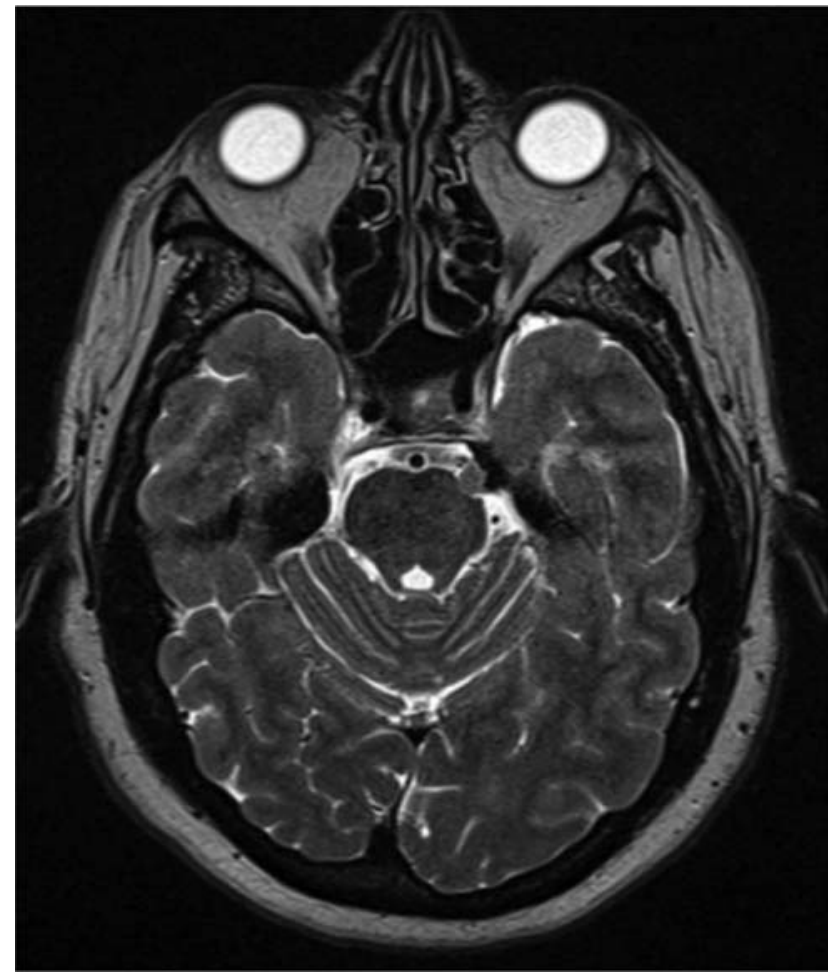

Fig. 1 Axial T2-weighted MRI image showing an 6- $\times$ 8-mm mass present on the left trigeminal nerve, determined by radiologists to be a trigeminal schwannoma. This image was taken before CyberKnife treatment, although subsequent imaging showed no changes in the mass.

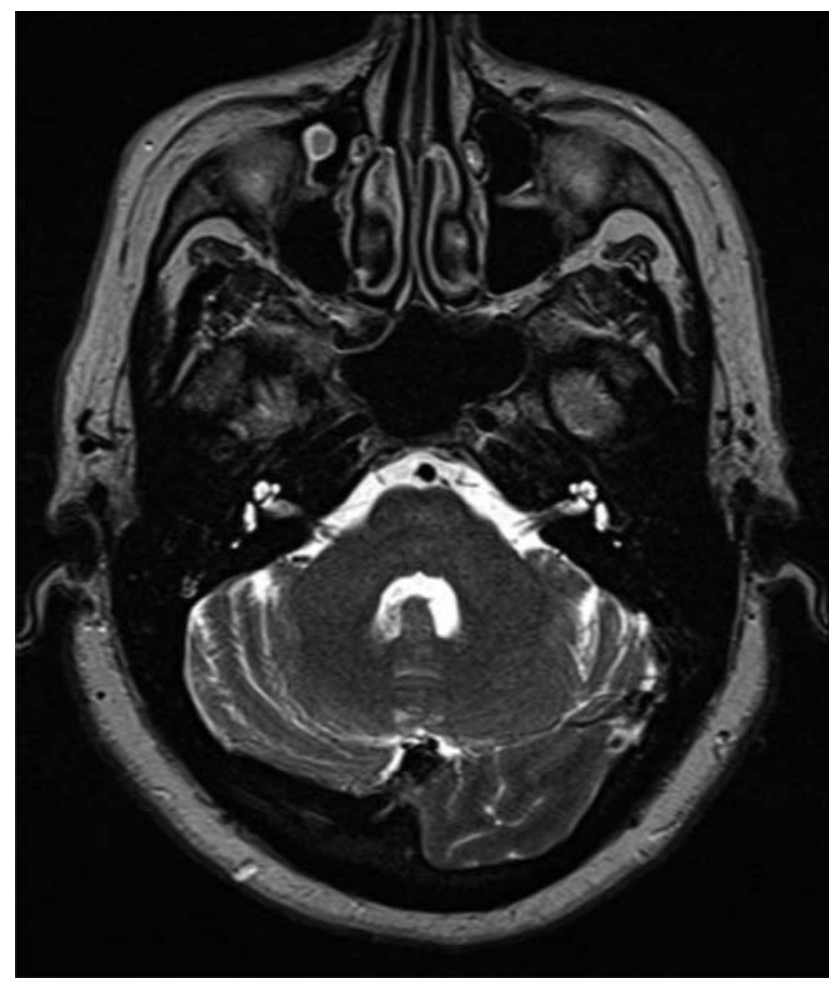

Fig. 2 Axial T2-weighted MRI image showing normal vestibulocochlear nerves bilaterally, without visible external compression, edema, or masses. This image was also taken before CyberKnife treatment, although subsequent imaging showed no changes in this area. 
However, the physicians recommended early treatment before potential tumor growth and symptom onset, as tumor invasion into nearby structures may be difficult to reverse. The risks, benefits, and alternatives of surgical resection and of stereotactic radiosurgery were discussed with the patient. She opted to undergo stereotactic radiosurgery because of the relatively minimal associated risks and a desire for early treatment of the tumor.

CyberKnife was chosen as the stereotactic radiosurgery technology, and the patient was dosed with $21 \mathrm{~Gy}$ in three fractions. After CyberKnife treatment, a followup brain MRI a few months later showed no change in the trigeminal schwannoma and no evidence of edema or radiation change existed around the trigeminal or vestibulocochlear nerves. The patient was never treated for her initial vestibulocochlear symptoms, which remained persistent throughout the year while being treated for her asymptomatic trigeminal schwannoma.

\section{Medical Record Keeping}

Medical records seemed to contradict the current diagnosis, as one of the many physicians involved in this case had mistakenly confused the trigeminal schwannoma with an acoustic neuroma. Subsequent notes autopopulated with acoustic neuroma and 10th revision of the International Statistical Classification of Diseases and Related Health Problems (ICD-10)-D33.3 to perpetuate this false diagnosis and confuse future providers. On further investigation, only the trigeminal schwannoma was confirmed radiographically, and the patient's diagnosis was reaffirmed.

\section{Symptom Persistence}

Two months after the second MRI, the patient's dizziness and left-sided hearing loss progressed to such a severity that she sought relief at an urgent care center where was told she had "fluid behind the ear." The patient was also experiencing leftsided ear pressure and pain, disequilibrium, and nausea. She was prescribed a weeklong regimen of dexamethasone ( $2 \mathrm{mg}$ twice per day) and followed up with radiation oncology. The oncologist referred her to an otolaryngologist for further evaluation of vestibulocochlear symptoms and otologic examination findings.

One month later, she presented to the current otolaryngologist with the symptoms she had experienced at the urgent care center, showing little improvement after the dexamethasone regimen. The patient's additional past medical history revealed omeprazole as her only current medication, taken for gastroesophageal reflux disease, and noncontributory surgeries more than three years before the symptom onset. Family history was considered insignificant. The patient denied tobacco, alcohol, or recreational drug use. Review of systems was negative for recent headaches, blurry vision, diplopia, facial pain, facial numbness, paresthesias, dysesthesias, weakness, falls, seizures, syncope, memory loss, changes in lacrimation or salivation, weight changes, palpitations, shortness of breath, changes in bowel habit, changes in urinary habits, or new skin lesions.

\section{Physical Examination}

Physical examination showed an obese, well-appearing female in no acute distress. She was normocephalic, atraumatic, and her neck was supple. No lymphadenopathy or thyroid nodules were felt on palpation. On otoscopic examination, the ear canals were clear without otorrhea or cerumen present, and tympanic membranes were transparent with normal light reflex bilaterally. Tympanometry was performed by an audiologist and showed Type A tympanograms bilaterally, suggesting normally functioning tympanic membranes. An audiological evaluation was then performed, which revealed a combined low- and high-frequency sensorineural hearing loss of 30-60 dB for the left ear, and normal hearing for the right ear (see - Figure 3 ). Videonystagmography (VNG) revealed $26 \%$ caloric weakness for the left ear, with a directional preponderance of $42 \%$ to the left. Cold-water irrigation evoked a weaker response in the left ear $\left(11^{\circ} /\right.$ second $)$ than the right ear $\left(33^{\circ} /\right.$ second $)$, and responses to warm-water irrigation were also asymmetric $\left(13^{\circ} /\right.$ second for the left ear and $8^{\circ}$ /second for the right ear) (see - Figure 4). Dix-Hallpike testing did not produce dizziness or visible nystagmus. No acoustic reflex testing was performed because of
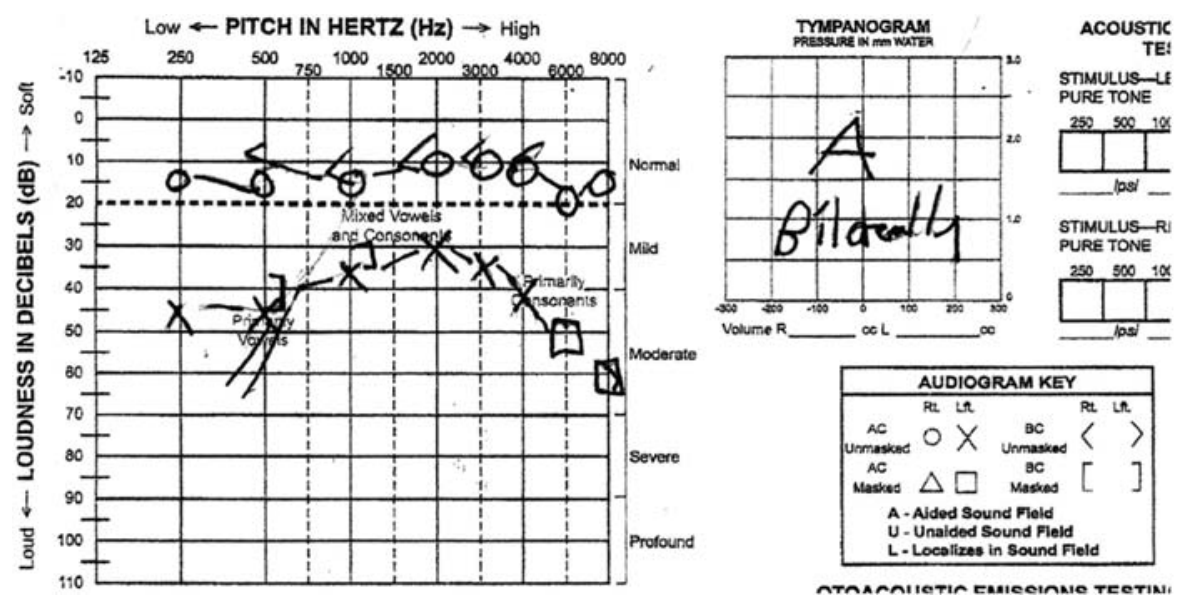

Fig. 3 Audiogram showing a left-sided, 30-dB combined low- and high-frequency sensorineural hearing loss, with normal hearing on the right side. Tympanometry showing Type A bilaterally, indicating normal tympanic membrane function. 


\section{Bithermal Caloxic}
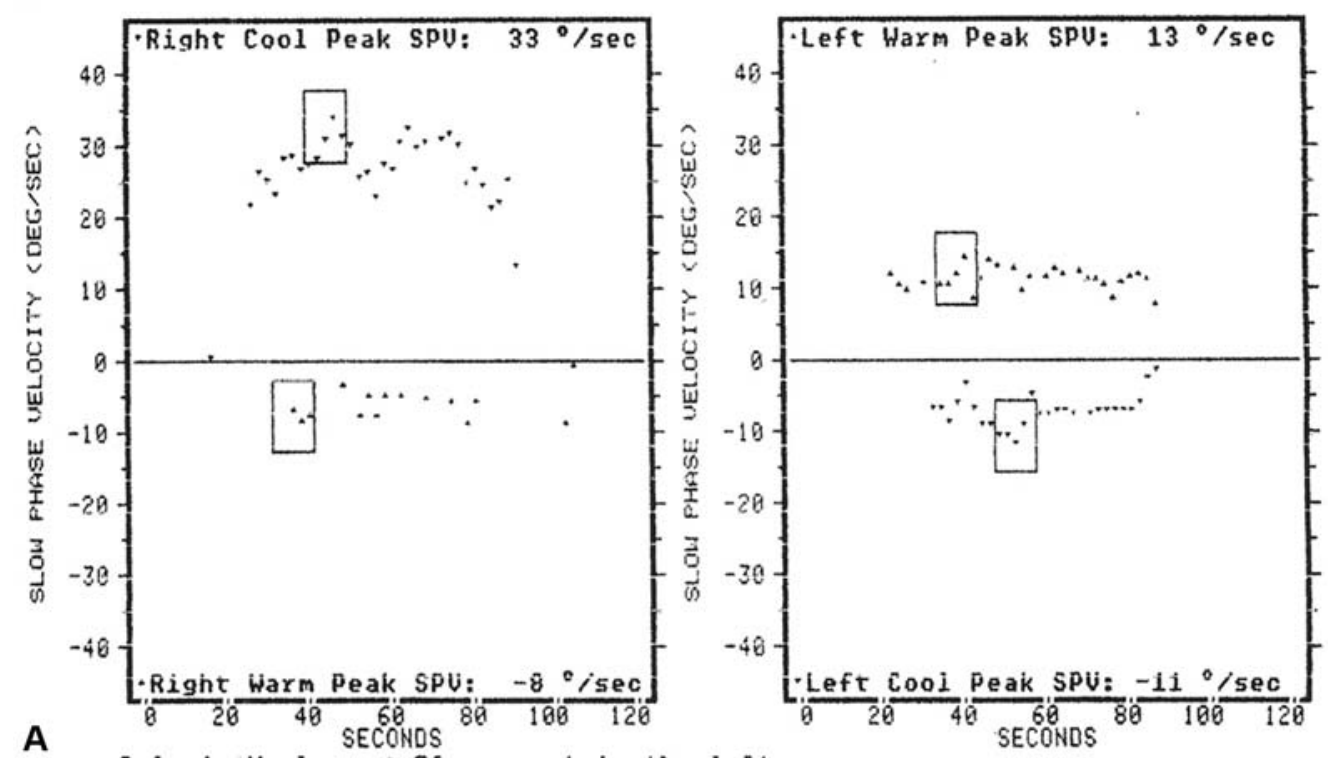

Caloric Weakness: 26 percent in the left ear

Directional Preponderance: 42 percent to the left

\section{Butterfly Chart}

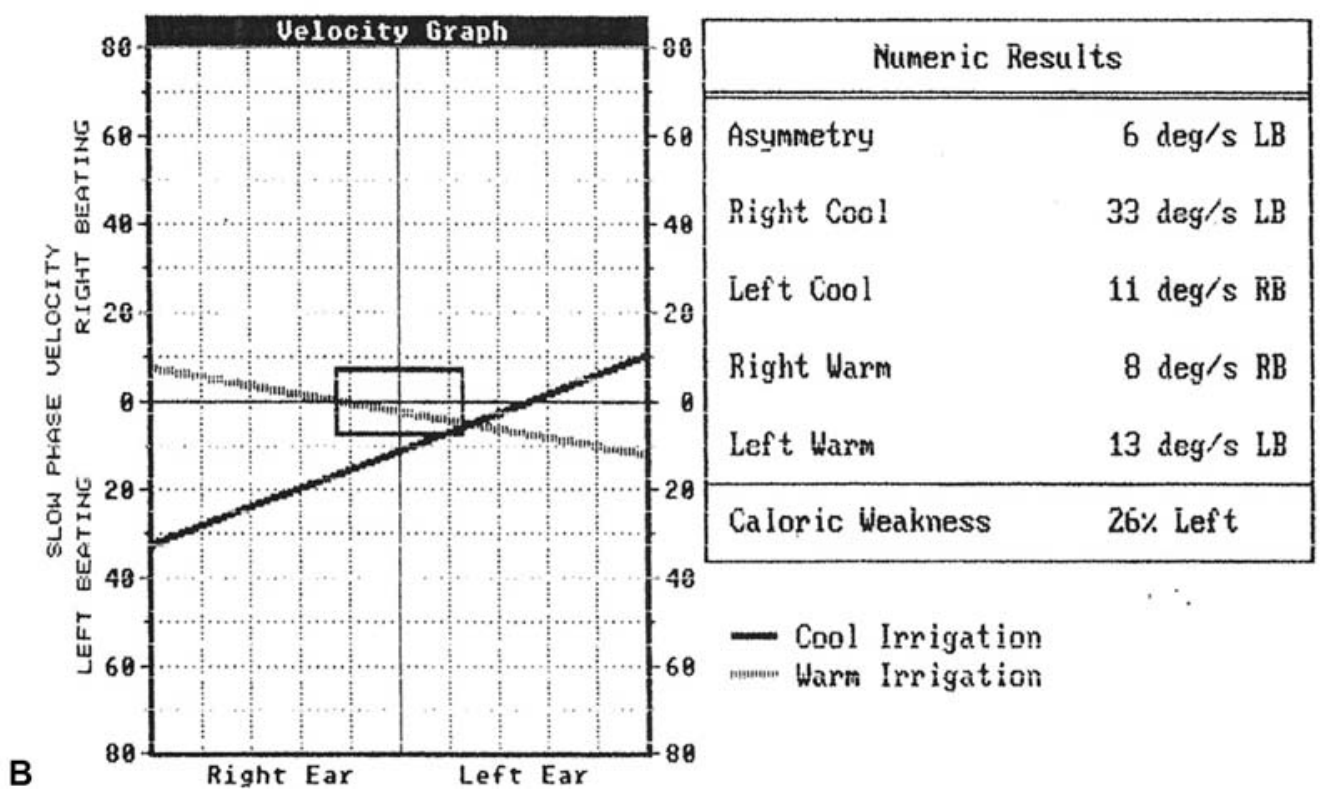

Fig. 4 VNG revealing ipsilateral left peripheral vestibular weakness, which was statistically significant->25\%, shown in (A) with bithermal caloric testing. A corresponding butterfly chart with asymmetry is shown in (B).

diagnostic confidence in a peripheral etiology. Sensation was intact and equal bilaterally in the distribution of the ophthalmic, maxillary, and mandibular branches of the trigeminal nerve. No mandible weakness or deviation was noted.

\section{Follow-Up}

At the otolaryngologist's office, the patient was given a 30-day oral prednisone taper and was instructed to return in one month. During this follow-up visit, the patient reported significant symptom improvement in dizziness, hearing, and aural fullness. Repeat audiological evaluation showed an improve- ment in the left-ear hearing at $250,500,4000$, and $5000 \mathrm{~Hz}$, although hearing in this ear remained mildly impaired with a 30- $\mathrm{dB}$ sensorineural hearing loss. She was given another prescription of steroids, and at the subsequent follow-up visit, she did not show further improvement in her audiologic symptoms. Most recently, she was fitted for a hearing aid, and she reported good aided benefit with improved communication ability. She was prescribed a diuretic, a benzodiazepine as needed for dizziness, and advised to follow a low-salt diet for suspected Meniere's disease. She will continue to be followed by the otolaryngologist for monitoring and treatment 
of vestibulocochlear symptoms. The radiation oncologist will continue to order periodic brain MRI scans every six months (or as needed) to monitor progression of the trigeminal schwannoma and any signs of trigeminal nerve compression.

\section{Discussion}

Decreased hearing has been reported rarely with trigeminal schwannomas, but only in addition to other symptoms of trigeminal nerve dysfunction (Fukaya and Yoshida ${ }^{6}$ ). It is peculiar that this patient did not show any of the usual signs or symptoms of trigeminal nerve dysfunction, such as ipsilateral facial pain or numbness. The radiologist, radiation oncologist, and otolaryngologist agreed that the patient's trigeminal schwannoma was an incidental finding and unrelated to her vestibulocochlear symptoms.

\section{Differential Diagnosis}

This patient's vestibulocochlear symptoms were most likely due to a peripheral etiology such as Meniere's disease, although diagnostic consideration must be given to viral or autoimmune conditions, Eustachian tube dysfunction (ETD), and other internal ear disorders (Lopez-Escamez et $\mathrm{al}^{9}$ ). Ipsilateral unilateral caloric weakness via VNG suggests a peripheral vestibular disturbance such as Meniere's disease, rather than a central one (McCaslin et $\mathrm{al}^{10}$ ). Furthermore, acoustic reflex testing on initial or follow-up evaluation may have indicated a peripheral etiology, although testing was not completed at either instance because of diagnostic confidence. Weighing the patient's two or more episodic symptoms, favorable response to corticosteroids, and the relatively common nature of the disease, the otolaryngologist concluded that the most likely diagnosis was Meniere's disease. ETD can also present with similar symptoms, show improvement with corticosteroids, and is relatively common. The patient showed Type A tympanograms, arguing against ETD, although data on utility of tympanograms for ETD alone are limited (Smith and Tysome ${ }^{15}$ ). Infectious causes such as viral cochleitis or labyrinthitis were considered less likely because of the prolonged and episodic nature of the patient's symptoms. Autoimmune inner-ear disease also improves with corticosteroids, although usually presents bilaterally (Harris et $\mathrm{al}^{8}$ ). The Dix-Hallpike test, the act of rapidly moving the patient's head from a straight position to a "head-hanging" position, suggested against benign paroxysmal positional vertigo (Viirre ${ }^{16}$ ). Medications, substances, and previous illnesses were unlikely contributors to the patient's presentation. The patient's initial presentation one year before included hearing loss, but not left-sided aural fullness, suggesting a recent disease flare before presenting to the otolaryngologist. Meniere's disease can episodically increase in severity.

Etiologies proximal to the internal ear must also be considered for this aberrant patient presentation. It is not uncommon for patients to have multiple schwannomas concurrently (Javalkar et $\mathrm{al}^{7}$ ). There exists the possibility that another schwannoma within her vestibulocochlear nerve, unidentifiable by brain MRI, may have been causing her vestibulocochlear symptoms. It is also possible that the trigeminal schwannoma seen on imaging could have com- pressed the vestibulocochlear nerve or vestibular/cochlear brainstem nuclei, although the MRI scans did not show direct compression of these structures. Although biopsies of the tumor were never performed, radiologic evidence based on tumor characteristics and location was sufficient for the diagnosis of trigeminal schwannoma. This tumor was of small size in comparison with other trigeminal schwannoma case reports in which patients were symptomatic. Vestibular migraine must also be considered for this patient, which may occur concurrent with Meniere's disease, although the patient denied headaches. Transient ischemic attacks are considered, but less likely for this young patient without significant cardiovascular risk factors. Although there was a lack of focal neurologic symptoms, cases of isolated vestibular symptoms from transient ischemic attacks have been reported (Lopez-Escamez et $\mathrm{al}^{9}$ ).

\section{Treatment Choice}

Although the treatment of choice for trigeminal schwannomas is surgical resection, the patient deemed the risks too high for treatment of an asymptomatic tumor. Stereotactic radiosurgery with CyberKnife did not immediately affect the patient's tumor, and her MRI was virtually unchanged after treatment, although not increased in size. According to the radiation oncologist, further treatment with this technique is no longer an option for this patient, as she already received the maximal radiation dose. MRI at one and two year followup revealed minimal tumor regression. Surgery may be indicated if the tumor progresses further.

\section{Diagnostic Confusion}

Many in medicine commonly regard the term intracranial schwannoma to be synonymous with acoustic neuroma. However, intracranial schwannomas encompass not only acoustic neuromas but also rare Schwann cell tumors, including trigeminal schwannomas. In addition to acoustic neuroma being a misnomer in and of itself, this inconsistency in nomenclature could easily lead physicians astray from a correct diagnosis. Autopopulation of previous diagnoses and a requirement to assign ICD-10 codes may have also contributed to a wrongly documented and perpetuated diagnosis. ICD-10-D33.3 represents a "benign neoplasm of cranial nerves," which includes all types of cranial nerve schwannomas (Centers of Disease Con$\operatorname{trol}^{3}$ ). A single code like this could further encourage the idea that schwannomas are of a single type-the acoustic neuroma. This case was further complicated by the fact that the patient's clinical presentation was suspicious for an acoustic neuroma, leading to a logical cascade of errors. When referring to schwannomas, specific terms such as vestibular schwannoma and trigeminal schwannoma exhibit a clarity and understanding that the term acoustic neuroma lacks.

\section{Conclusion}

Trigeminal schwannomas are exceedingly rare tumors that usually present with symptoms of trigeminal nerve dysfunction, such as a facial pain, numbness, or paresthesia in the distribution of the trigeminal nerve unilaterally. However, 
this patient presented with solely unrelated vestibulocochlear symptoms, which were falsely suggestive of a vestibulocochlear schwannoma. This tumor was misdiagnosed in a physician note, leading to confusion in subsequent otolaryngology and radiation oncology visits.

From this case, we can see that imaging does not always concur with symptoms and that multiple etiologies can confuse a clinical picture. Rare diagnoses can be mistaken for their relatively more common counterpart, and special attention must be taken to examine the whole clinical picture and to keep the medical record consistent. In the future, an emphasis on a more accurate approach to rare diagnoses must be made to minimize potentially life-threatening mistakes. It is insufficient to rely on imaging or clinical symptoms alone when interpreting patient presentations and prior physician documentation, as it can lead to a false diagnosis and delayed treatment.

\section{Conclusion}

Based on the results of this study, nursing knowledge of capillary refill, venous congestion, and basic microsurgical free flap physiology is inadequate. With implementation of a teaching in-service curriculum highlighting these key areas of deficiencies, nurses improved both their understanding and confidence levels, regardless of their level of experience. This has the potential to improve recognition of flap compromise, the first step in increasing flap survival rates.

\section{Abbreviations}

$\begin{array}{ll}\text { CT } & \text { computerized tomography } \\ \text { ETD } & \text { Eustachian tube dysfunction } \\ \text { Gy } & \text { gray } \\ \text { ICD-10 } & \begin{array}{l}\text { 10th revision of the International Statistical Clas- } \\ \text { sification of Diseases and Related Health Problems }\end{array} \\ \text { MRI } & \text { magnetic resonance imaging } \\ \text { TIA } & \text { transient ischemic attacks } \\ \text { VNG } & \text { videonystagmography }\end{array}$

Conflict of Interest

None declared.

\section{Acknowledgments}

We acknowledge Nevada ENT for allowing us to use their medical record database and for their continued support in medical education. A previous version of this report was presented at the American College of Physicians regional abstract competition in 2017 by Lauran Evans.

\section{References}

1 Agarwal A. Intracranial trigeminal schwannoma. Neuroradiol J 2015;28(01):36-41

2 Arseni C, Dumitrescu L, Constantinescu A. Neurinomas of the trigeminal nerve. Surg Neurol 1975;04(06):497-503

3 Center of Disease Control. FY 2018 CD-10-CM. https://www.cdc. gov/nchs/icd/icd10cm.htm. Accessed February 8, 2018

4 Chowdhury FH, Haque MR, Kawsar KA, Sarker MH, Hasan M, Goel $\mathrm{AH}$. Intracranial nonvestibular neurinomas: young neurosurgeons' experience. J Neurosci Rural Pract 2014;5(03):231-243

5 Coelho DH, Lalwani AK. Medical management of Meniere's disease. Laryngoscope 2008;118:1099-1108

6 Fukaya R, Yoshida K. Trigeminal schwannomas: experience with 57 cases and a review of the literature. Neurosurg Rev 2010; 34:159-171

7 Javalkar VK, Pigott T, Pal P, Findlay G. Multiple schwannomas: report of two cases. Eur Spine J 2007;16(03):287-292

8 Harris DA, Mikulec AA, Carls SL. Autoimmune inner ear disease preliminary case report: audiometric findings following steroid treatments. Am J Audiol 2013;22(01):120-124

9 Lopez-Escamez JA, Carey J, Chung WH, Goebel JA, Magnusson M, Mandaia M, Newman-Toker DE, Strupp M, Suzuki M, Trabalzini F, Bisdorff A; Classification Committee of the Barany Society; Japan Society for Equilibrium Research; European Academy of Otology and Neurotology (EAONO); Equilibrium Committee of the American Academy of Otolaryngology-Head and Neck Surgery (AAOHNS); Korean Balance Society. Diagnostic criteria for Meniere's disease. J Vestib Res 2015;25:1-7

10 McCaslin DL, Rivas A, Jacobson GP, Bennett ML. The dissociation of video head impulse test (vHIT) and bithermal caloric test results provide topological localization of vestibular system impairment in patients with "definite" Meniere's disease. Am J Audiol 2015;24 (01):1-10

11 Pearson BW, Brackmann DE. Committee on hearing and equilibrium guidelines for the evaluation of hearing preservation in acoustic neuroma (vestibular schwannoma): committee on hearing and equilibrium. Otolaryngol Head Neck Surg 1995;113(03):179-180

12 Rigamonti D, Spetzler RF, Shetter A, Drayer BP. Magnetic resonance imaging and trigeminal schwannoma. Surg Neurol 1987;28 (01):67-70

13 Sajjadi H, Paparella MM. Meniere's disease. Lancet 2008;372 (9636):406-414

14 Sharma BS, Ahmad FU, Chandra PS, Mahapatra AK. Trigeminal schwannomas: experience with 68 cases. J Clin Neurosci 2008;15 (07):738-743

15 Smith ME, Tysome JR. Tests of Eustachian tube function: a review. Clin Otolaryngol 2015;40(04):300-311

16 Viirre E, Purcell I, Baloh RW. The Dix-Hallpike test and the canalith repositioning maneuver. Laryngoscope 2009;115(01):184-187

17 Zhang L, Yang Y, Xu S. Trigeminal schwannomas: a report of 42 cases and review of the relevant surgical approaches. Clin Neurol Neurosurg 2009;111(03):261-269 